\title{
Detailed Requirements for Robots in Autism Therapy
}

\author{
Nicole Giullian, Daniel Ricks, Alan Atherton, Mark Colton, Michael Goodrich, Bonnie Brinton \\ Brigham Young University \\ Provo, UT, United States \\ nicole.giullian@gmail.com,danjricks@gmail.com, jalanatherton@gmail.com, colton@byu.edu, \\ mike@cs.byu.edu, bonnie_brinton@byu.edu
}

\begin{abstract}
Robot-based autism therapy is a rapidly developing area of research, with a wide variety of robots being developed for use in clinical settings. Specific, detailed requirements for robots and user interfaces are needed to provide guidelines for the creation of robots that more effectively assist therapists in autism therapy. This paper enumerates a set of requirements for a clinical humanoid robot and the associated human interface. The design of two humanoid robots and an intuitive and flexible user interface for use by therapists in the treatment of children with autism are described.
\end{abstract}

Keywords-Autism, robotics

\section{INTRODUCTION}

Children diagnosed with autism typically display difficulty in the areas of social interaction, communication, and repetitive behavior. The inability to establish and maintain joint attention, which is the ability of interactional partners to share a focus of interest, also constitutes a core deficit in children with autism. This deficiency is pervasive and is associated with problems in later language, cognitive, and social development [1]. Despite its importance, interventions focused on the development of joint attention in children with autism are relatively rare and have produced varying levels of success.

Recent research suggests that robots may be used effectively as tools to improve joint attention skills for children with autism. Robots are highly engaging to these children and have been shown to elicit behaviors that may not often be seen in child-person interactions. Promising methods have shown that the child's natural interest in robots can encourage communication with the therapist [2]. One of the challenges, however, is to help children with autism generalize interactional skills from robots to humans, a connection that is often very difficult to establish. Our research is designed to establish three-way interactions between the child, clinician, and robot in an effort to help the child generalize joint attention from robot-child interactions to clinician-child and parent-child interactions.

Researchers have created a wide variety of robots in an effort to provide effective tools to therapists. Both researchers and therapists use their best judgment to try to predict a robot's performance in therapy, but this type of assessment is somewhat subjective. Detailed requirements for robots could provide another means to predict the effectiveness of a robot in autism therapy. The purpose of this paper is to develop detailed requirements for robots and user interfaces to maximize the usefulness of robots as therapeutic tools.

\section{A. Related Work}

In recent years researchers have begun to explore the use of robots to achieve specific therapeutic objectives for children with autism. Although robots and other technologies have been suggested as tools to aid in the early diagnosis of autism [4,5], the majority of research focuses on developing robots and novel therapies that will help alleviate symptoms in children that have been previously diagnosed. During initial research involving robot-based therapy, there has been anecdotal evidence that children with autism exhibit less severe autistic behavior when interacting with robots than when interacting with their peers [6]. Some therapies have included helping the child to improve their self-initiated interactions, turn-taking skills, imitation abilities, emotion recognition, and joint attention abilities [7]. These behaviors have been encouraged by having the robot react to the child's actions. Playing chase games with the child [8] or blowing bubbles when the child presses a button on the robot [9] are examples of such activities. Other activities involve asking the child to mimic the robot's actions [10,11], to identify the emotion a robot's face is displaying $[11,13]$, or to look in the direction the robot points [14]. In some activities the child directly interacts with the robot by him/herself with a parent or clinician on hand to help encourage this interaction $[13,14]$, while in other scenarios a therapist is "in-the-loop" and plays a more active part in the therapy $[15,16]$. A therapist in the loop could either remotely control the robot for enhanced robot-child interaction, or could be in the room with the child and robot so that the robot's presence enhances the therapist-child interaction.

The robots used in these therapies vary greatly. It has been observed that children with autism tend to favor robots that do not too closely resemble a real person [17]; however, naturalistic approaches to autism therapy suggest that the more closely a clinical setting approaches the real world, the more likely the child will be able to generalize what is learned in the clinic to interactions outside the clinic [20]. Thus, it is possible that a robot with a realistic human appearance could improve the chances of generalization. This has led some to suggest that a series of increasingly realistic robots, or a robot that can progressively change its appearance, could lead to the greatest therapeutic benefit $[5,17]$.

To address the issue of generalization, some researchers have developed humanoid robotic platforms that are extremely lifelike while others have built robots that do not have a humanoid appearance. Researchers at the University of Hertfordshire, for example, have used non-humanoid, mobile robots; a doll-like robot, Robota; and a humanoid, child-sized 
robot, KASPAR, in their autism studies [8,17]. Researchers at Yale have used a dinosaur robot, Pleo [5], and researchers in Japan have used a mechanical, machine-like robot, Infanoid, as well as a small, snowman-looking robot, Keepon [18]. Researchers at the University of Pisa have used a realistic robot, FACE [13], whereas researchers at the University of Sherbrook and Toyota have used less realistic humanoid robots in their studies, such as Tito and HOAP-3 $[11,14]$.

\section{B. Robot Specifications}

Many advances have been made in the use of robots in therapy of children with autism, and the development of detailed requirements has the potential to help predict and improve upon the effectiveness of clinical robots for use in the treatment of children with autism. As mentioned previously, a wide variety of robots have been created with great variations in shape, size, and style. The evaluation of their effectiveness is primarily based on the judgment and experience of expert clinicians and engineers.

It has been suggested that a robot must be robust, easily reprogrammable, affordable [16], and appealing to children with autism in order to be useful in therapy. Other requirements that have been proposed for a robot include having aspects familiar to the child, providing choices, having a modular design that can easily be customized, being simple in appearance, and able to be manipulated by the child $[3,12]$.

The ultimate goal of using robots in autism therapy is to increase the effectiveness of certain types of clinical treatments; establishing detailed requirements for both the robots and the interface to control the robots could help to assess the effectiveness of existing robot systems and to improve the design of future systems. Including expert therapists and autism researchers in the development of these requirements is an essential element, because they ultimately must judge the clinical benefits of using these technologies in the clinic. Furthermore, it is essential to create technologies that are practical in a clinical setting, which requires that the robots are robust, useable, versatile, and safe. Clinicians and autism researchers are again the ultimate judges of a robot system's success in these areas

This paper proposes a set of requirements that will aid engineers in creating robots and interfaces that will be effective and practical for use in autism therapy.

\section{THERAPEUTIC MODEL}

Robots may be used both to prime social responses from the child as well as to help the child apply these responses to the therapist. A number of protocols are being developed to effectively apply the robot in this manner.

\section{A. Purpose of the Robot}

One of the major purposes of a robot in autism therapy is to help teach children with autism appropriate social responses and create situations in which children can practice these skills. The use of a robot in therapy may be able to prime social responses that otherwise would not be possible.

A second purpose of the robot is to help the children apply the social responses learned from the robot to interactions with the therapist and, ultimately, with family and peers outside the clinic. Interaction with the therapist may prove to be a critical step in assisting the child to generalize the skills learned with the robot to interactions with other people. Standard therapies between a therapist and a child on the autism spectrum have been used for many years with varying levels of success in helping the children to generalize their skills learned in the clinic. By using the robot to help encourage this child-clinician interaction, it is hoped that the level of transfer will also be enhanced. To be able to generalize effectively, children often need repeated practice, and the repeated practice of responding to the robot's and therapist's actions in the clinic is meant to help prepare a child with autism to generalize in other situations.

To be effective, robots should be integrated smoothly into therapy sessions. In the example therapies described in the next section, two clinicians work together: a primary clinician and an assistant. The primary clinician performs gestures and interacts with the child, while the assistant helps the child imitate actions and helps control the child in case of erratic or violent behavior. Although the benefits may be significant, the use of robots may complicate therapy situations and place additional demands on the therapist. In therapies that involve the child, robot, and clinician, the clinician must not only interact with the child and execute effective therapeutic techniques, but also control the actions of the robot. For this reason, traditional therapeutic techniques must be modified and adapted to effectively incorporate a robot. This also emphasizes the need to develop robotic systems that can be used effectively by the therapists without hindering the therapeutic objectives.

\section{B. Therapeutic Activities}

In the proposed model, interventions consist of robot-based activities to encourage joint attention between child and clinician. Thus, for each activity the robot is used as a "partner" or facilitator for social interaction between the child and the clinician. The efficacy of the robot depends greatly upon its ability to be used by the therapist to elicit a triadic, or threeway, interaction. There is a clear goal for each activity and a series of steps where the clinician references the robot's actions in an attempt interact with the child. These activities were developed by experienced therapists at the BYU Comprehensive Clinic, and two examples are listed below.

In the first activity, the clinician uses the robot to emphasize reactions to success or failure of an action with an appropriate positive or negative emotion. The child is assisted to imitate the clinician's actions and react differentially to the success of the action. The procedure develops as follows:

1. Clinician successfully performs a gesture (e.g., puts hands up, beats on a drum, or offers a snack) and reacts with positive emotion ("Up! Hah!”)

2. Robot performs same successful action

3. Robot reacts positively (light/sound/motion)

4. Clinician reacts to the robot's action with positive emotion ("Wow!")

5. Clinician prompts the child to perform the action

6. Assistant helps child (hand-over-hand) to perform the same action 
7. Clinician reacts positively to the child ("You did it!")

8. Robot reacts positively to the child (light/sound/motion)

9. Sequence is repeated with different tasks

10. At occasional intervals, clinician attempts actions and fails (puts only one hand up part way, misses the drum, drops the snack)

11. Clinician reacts with negative emotion ("Phooey!")

12. Robot reacts negatively to the clinician's failure (sound)

13. Clinician repeats task successfully

14. Clinician and robot react with positive affect

15. At occasional intervals, robot attempts action and fails

16. Robot reacts negatively (sound)

17. Clinician reacts negatively (" $\mathrm{Oh}$, phooey!)

18. Sequence is completed with successful actions and positive affect

19. If child is unsuccessful with hand-over-hand action, clinician reacts sympathetically with negative emotion

20. The robot also reacts negatively to the child's failure (sound)

Joint attention will be encouraged as the therapist reacts to the actions of the robot. The mistakes of the robot will also provide an opportunity for the child to share interest or excitement with the therapist.

The second activity involves a give and take reciprocal action with the robot, again using the robot to emphasize reactions. This turn-taking activity has the goal of eliciting joint attention between the child and the therapist, possibly as the child makes mistakes or as the robot makes mistakes.

1. Clinician hits tambourine and reacts positively

2. Robot reacts positively (sound/light)

3. Clinician positions tambourine under the robot's arm and prompts it ("Your turn!")

4. Robot hits the tambourine and reacts positively (sound/light)

5. Clinician reacts positively ("You did it!")

6. Clinician positions tambourine under the child's hand and prompts him ("Your turn!")

7. Assistant helps the child hit the tambourine

8. Clinician reacts positively ("You did it, too!")

9. Robot reacts positively (light/sound)

10. At occasional intervals the robot attempts to hit the tambourine and misses

11. The robot responds negatively (light/sound)

12. Clinician reacts with negative emotion ("Oh no!")

13. If child is unsuccessful with hand-over-hand action, clinician reacts sympathetically with negative emotion

14. Robot also responds negatively (light/sound)

These therapy outlines have simple if-then and do-while logic, making them straightforward both for the therapists and for the designer and engineers who are building the robot and programming its actions.

\section{The Need for Specifications}

Using robots in therapy in the aforementioned manner could improve the efficacy of therapy. Detailed design specifications would also be helpful to develop new robots and predict which robots will be more effective in therapy. For example, a robot that is not easily controlled or that is too complex may complicate the therapy rather than make it more effective. In any design process, specifications and requirements are needed to set constraints and design objectives. Without such requirements, it is difficult to assess the efficacy of a robot design; trial-and-error may be used, but it is a costly approach that requires significant time to implement. A wide variety of robots could be created for use in therapy, but providing a specific set of requirements for such a robot will provide a means to predict the effectiveness of a robot in therapy. This will in turn help designers improve robots and interfaces, thus resulting in superior designs.

\section{RoBot DESIGN REQUIREMENTS}

Each type of robot has differing strengths and weaknesses, but the most important requirement for the robot is that it be able to perform the desired activities designed by therapists. Since children may interact with humanoid robots in similar ways as they interact with other people, this paper focuses on the design of humanoid robots. The ability to perform the desired activities can be broken down into three categories of more objective requirements: functionality and appearance, safety requirements, and autonomy.

\section{A. Functionality and Appearance}

The functionality and appearance of the robot will have a strong influence on its effectiveness in therapy for children with autism. Five important requirements that were developed in consultation with clinicians are listed below.

First, the robot must be visually engaging to a child. A careful balance must be found here, because a robot with too many colors or with too great of complexity may over stimulate the child with autism and have an adverse effect. The robot should have a neutral-colored torso, a face with distinct features, and hands that are a distinct color. Particular emphasis should be placed on the face of the robot, because some therapies involving the robot could be to help the child to understand facial cues and connect them with emotions. A simpler format, particularly for the face, will help prevent overstimulation or confusion.

Second, the appearance of the robot should not be overly realistic, because making the robot too human-like may reduce the interest of the child [17]. Maintaining a somewhat mechanical or mascot appearance is important to spark the child's interest, but the robot should not be too mechanical or the child may be more interested in examining its components than in interacting with the robot [18]. The robot should be easily discernable as humanoid, but with enough mechanical properties to be recognizable as a robot.

Third, the size of the robot should be roughly the size of a human toddler. This requirement, determined in conjunction 
with therapists, was specified for a variety of reasons [2]. The target audience for therapy is children, particularly younger children, so the robot will be approximately the same size as many of the clients it will help. Similarity in size will make the robot less intimidating and hopefully more interesting to the child. Being approximately the same height as the robot will allow the child to be at eye-level with the robot. If a child is able to practice interaction with a robot of about his own size, generalization of skills to other children may be easier. In addition, imitation of a robot of similar size may be more intuitive than imitating an extremely large or small robot.

Fourth, the range of motion and degrees of freedom of the robot should be similar to those of a human toddler. This will enhance the feeling that the robot is much like the toddler, and more importantly, allow it to perform the required actions. To imitate the movement of a toddler, the robot would ideally have four degrees of freedom per arm: three at the shoulder (abduction/adduction, flexion/extension, and humeral rotation) and one at the elbow. Many activities would be possible with only three degrees of freedom, but this would limit the types of possible motions. Joint limits should be set to prevent positions impossible for humans, such as an overextended elbow.

Fifth, the robot must be strong enough to move small objects. These objects may be toys, such as blocks or balls, food, or other objects such as tambourines. Most objects the robot will need to move will be lightweight, so it does not need to exert large forces, but it must be able to consistently exert sufficient forces to move the objects.

\section{B. Safety Requirements}

Children with autism can be rambunctious and, if not carefully monitored, could be prone to touch or handle the robot, which could hurt the child or the robot. It is important to minimize pinch points. Fast, jerky motions could be dangerous if the child if is too close to the robot, so smooth, controlled motions are also preferred. In case of unexpected malfunctioning of the robot, an easily accessible emergency stop button should be included to allow the therapist to quickly deactivate the robot.

Of secondary importance is to keep the robot safe from the child. In order for the robot to be viable in a clinical setting, it must be able to withstand some mishandling as well as not cause harm to anyone else. The robot should be situated on a sturdy base so that it will not be easily knocked over, and the joints should be strong enough to withstand some mistreatment. The robot should also be designed to be modular and easily reassembled in case parts do break. Tactile interaction with the robot is important but should be kept under careful control; for added safety, therapists present in the room with the child and robot can prevent the child from roughly handling the robot.

\section{Autonomy}

The robot must have a certain level of autonomy [21], or the therapist's attention will be consumed in controlling the robot rather than in interacting with the child. A completely autonomous robot is not desired; some control by the therapist is important to allow the therapist to decide how the robot should respond, and whether the robot should advance to the next activity. The level of the child's interest, response to the robot, and interaction with the therapist are often factors that the robot cannot analyze. A human is needed in the loop to assess the state of the child and the progress of the therapy and to shape the robot's behaviors to maximize therapeutic impact. However, some autonomy is needed in the sequence of actions. In essence, the autonomy forms sets of behaviors that the therapist organizes, sequences, and manages to produce an effective therapy session. As a therapist plans a therapy session for a particular child, the robot will need to be able to store and execute choreographed motions. It must be able to execute a sequence of desired motions when indicated by the therapist. If the therapist has to control each individual motion as the therapy progresses, his or her attention will be diverted from the child. Choreographing the robot's sequences of motion ahead of time allows the therapist to focus more on the child during a therapy session.

\section{USER INTERFACE REQUIREMENTS}

The goal of the user interface is to make the smallest possible demand on the therapist during the robot's use in therapy. To accomplish this goal, the user interface controlling the robot must also meet certain requirements: it must be easily understandable to therapists, adaptable to sudden changes, and controlled with a handheld device.

\section{A. Understandable to Therapists}

In order to effectively use robots in therapeutic environments, interactive, triadic choreographies must be designed for the robot to follow, where the operator directs the robot in real time. A therapy session can be compared to a dance: the entire choreography is built from smaller dance moves. In the same way, choreography for therapy consists of several actions that the robot can perform, as well as actions for the therapist and child to perform. Choreography for therapy differs from dance in the sense that the therapist can direct the robot to change what actions it is performing at predetermined branching points to adapt to the child's actions. Choreography design could be done either by programmers or therapists.

We hypothesize that therapists need (a) to program robots to some extent and (b) should be able to direct the robot's programmed choreography during a therapy session. As explained by Yim, when an environment includes unpredictable elements, the primary users of a system must be able to program robots to adapt to novel situations [22]. In working with children with autism, there are many unpredictable events, both within an individual therapy session and over the course of several sessions (as the clinician tailors the therapy sessions to the needs and responses of the individual). Note that we do not believe it necessary for therapists to program robots during a therapy session, but there is potential benefit to having them program robots between therapy sessions. Although programmers could be called on to design new choreographies, enabling therapists to design choreographies without the help of a programmer will make the process more rapid and make the potential for therapy more powerful as the therapist is able to shape behaviors to maximize potential clinical benefit.

The challenge in enabling therapists to program the robot is that therapists rarely have training or experience programming 
robots or computers. This means we must design a user interface that allows therapists to program robots in a way that is understandable to them while still remaining flexible enough to use all of the desired features of the robots in use. Using certain programming methods will make the interface easy to use. Three potential programming methods are visual programming (also called graphical programming or dataflow programming), human motion capture with retargeting, and direct interaction.

Visual programming allows the expression of logical flow in a visual environment using graphical icons and connecting arrows to indicate program flow. Simple visual programming offers the power of a finite state machine, a machine that is powerful enough to capture the contingencies of a standard therapy session. Green and Petre evaluate two visual programming languages and conclude that visual programming is effective [23].

Human motion capture is one way of programming by demonstration. One could also manipulate the joints of the robot directly to capture a new action. While this is a useful and intuitive method for programming the robot, it requires additional sensors on the robot and requires the robot to be present. Motion capture allows a therapist to act out a desired action, after which motion retargeting attempts to construct a program for the robot to perform the same action. Motion capture needs a separate camera, but the camera does not need to be mounted on the robot, and the robot does not need to be present. One such system was developed by the Honda Research Institute to perform the motion capture and retargeting. Honda's system uses a time-of-flight ranging camera to capture a person's upper-body actions without the use of markers. The person needs only to stand at a particular distance from the camera and move not too quickly, and the system captures the actions with reasonable accuracy. Because the system is simple compared to solutions that use markers or special clothing, it seems better suited for novices to use. Once an action is captured, Honda's system can adapt the motions of the person to the robot's capabilities and constraints. The user interface could then include the action as one of the pieces to construct the entire choreography.

Direct interaction means the user can interact with graphical elements displayed in the interface directly, instead of using a secondary interface interaction element [24]. A graphical depiction of the robot could be displayed in the interface, and users could then click and drag the displayed components of the robot to move the virtual robot (Fig. 1). With this tool, users can create actions for the robot without needing to have the physical robot in proximity.

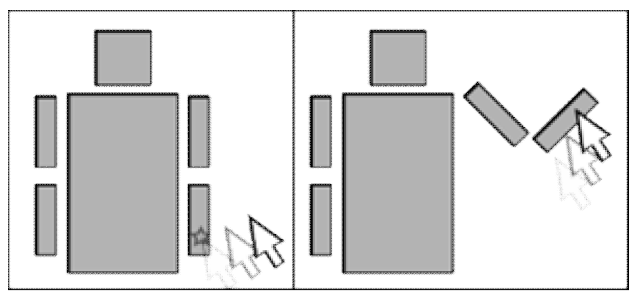

Figure 1. Sequence showing direct interaction with a user interface for a virtual robot

\section{B. Responsive and Flexible}

One option for using a robot in therapy is to design a choreography in its entirety beforehand and simply play back the entire choreography in a linear fashion. The primary drawback of such an approach is that children with autism will often not exhibit predictable patterns of interaction, losing interest in the robot for periods of time or perseverating on aspects of the robot that are not therapeutically productive. Without the ability to quickly change the behavior of the robot, it may be difficult to adapt the robot to a child's dynamic needs. A therapy choreography is essentially a state machine that allows the behavior of the robot to be modified at the therapist's command, producing a richer set of possible behaviors, all of which can be compatible with the objectives of a particular therapy session. In short, if a therapist sees an opportunity or need to modify the robot's behavior within a therapy setting, he must be able to quickly adapt.

\section{Control with a Handheld Device}

Because the therapist must interact with the robot during a therapy session, a user interface is needed. Workload on the therapist while interacting with a child is often extremely high, so the interface must increase the workload as little as possible. In addition, since children are often interested in electronic devices, the user interface must be discreet, allowing a therapist to hide or reveal the device as needed to facilitate productive interaction. Small devices can easily be concealed, but things like chorded keyboards or touch screen displays may be too complicated or require too much visual attention for use in a therapy session. A simpler solution is needed.

With these requirements, it seems that a reasonable interface is a small remote control (as for a television). Such a device could be concealed in a pocket or underneath a clipboard, as done by Scassellati [5]. Buttons on the remote control can be mapped directly to actions for the robot to perform when the choreography is simple enough to be represented by a few actions. This type of workload on the therapist ought to be low, since visual and auditory information is absent. However, when the choreography calls for numerous actions or branching points, a simple button-to-action mapping may not be able to represent all of the necessary choices. Instead, we can use a series of button pushes, much like dialing a phone number. Care must be taken, as good design of such an interface is more difficult and could lead to high workload.

\section{OUR RESEARCH}

Two robots, Troy and Trevor, and a user interface to control both of them have been created. These technologies have been developed with the objective of satisfying the requirements for use in therapy that were enumerated in previous sections.

\section{A. Troy}

Troy (Fig. 2) was designed to satisfy the aforementioned requirements. It is an upper-body, humanoid robot roughly the size of a four year old child. It is 25 " tall, and has two 12 " long arms, whereas an average four year old is 24.9 " tall from the crown of the head to mid-thigh and has 11.4" long arms from the shoulder to the wrist [19]. The arms have four degrees of freedom (DOF) each, including $2 \mathrm{DOF}$ in the shoulder for flexion/extension and abduction/adduction. The remaining 2 
DOF are for humeral rotation and elbow flexion/extension. These 4 DOF allow the robot to point in any direction and have a range of motion similar to that of a human.

Troy weighs $15 \mathrm{lbs}$ and sits on a 9" x 11" base. The base is large enough, and the robot heavy enough, to hold it in place without tipping over if it is lightly pushed, yet it is light enough to easily be moved around by a therapist.

The robot currently has over 30 pre-programmed actions available to a clinician to use in therapy settings. The actions are designed in to complete tasks that the clinicians deem useful. When a new therapy protocol is developed, the specific actions required to complete the protocol are programmed and made available for the therapists to use. Some actions include raising both arms straight up, using an arm to push an object, and bouncing its forearm to tap a tambourine or xylophone.

Since generalization of learned skills is so difficult to achieve, Troy has a computer screen for its face so that, as a child becomes more accustomed to Troy over time, the therapist could change faces to something more realistic. Children with autism tend to prefer less realistic robots, so Troy could start with a simple cartoonish face so that the child will not be intimidated. This face could eventually show the image of a human's face to achieve increased realism. The head is typically mounted horizontally (landscape) but can easily be mounted vertically (portrait) to have more humanoid proportions. The head is mounted on a 2-DOF pan-tilt neck.

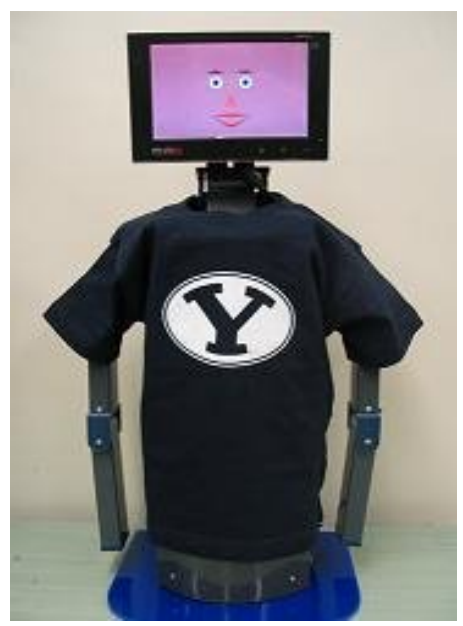

Figure 2. Troy

\section{B. Trevor}

Trevor (Triadic Relationship EVOking Robot) is a humanoid robot created using LEGO® Mindstorms (Fig. 3). The functionality and appearance of this robot closely matches the specifications listed above. It is strong enough to move objects and is about the size of a human toddler. Its shoulder only has two degrees of freedom (humeral motion is not available). This comes as a result of the limitations of LEGO® Mindstorms: the NXT bricks only control three motors at a time. The three degrees of freedom chosen were those deemed to be most important in the motion of the robot.

One of the strengths of this robot is that it is visually engaging to a child. Children's natural interest in LEGOs makes the robot interesting even before it begins to move. The face and hands are particularly interesting, and will hopefully draw the child's attention. Its mechanical appearance is enough to be interesting, but not so mechanical that the child would be overwhelmed.

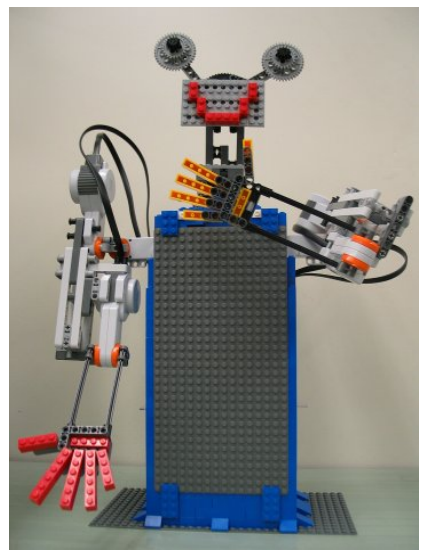

Figure 3. Trevor: A humanoid LEGO® robot

The benefit of using a LEGO® robot is that it can be easily reassembled if it is roughly handled by children. If anything were broken, the parts could be easily and inexpensively reassembled or, if necessary, replaced. TREVOR's movements are relatively smooth, and the motors do not provide enough force to pinch a child. In future revisions, motor wires will be covered to prevent the child from pulling them.

TREVOR is also autonomous to the degree necessary. Sequences of actions can be programmed and choreographed prior to therapy sessions.

\section{User Interface}

With suitable robots available, a user interface is needed for therapists to interact with the robot. The requirements shown in Table 1 are the guiding principles for designing the user interface.

TABLE I. USER INTERFACE REQUIREMENTS

\begin{tabular}{|l|l|}
\hline \multicolumn{1}{|c|}{ Requirement } & \multicolumn{1}{c|}{ Description } \\
\hline $\begin{array}{l}\text { Understandable to } \\
\text { therapist }\end{array}$ & $\begin{array}{l}\text { Need intuitive way to program robots for non- } \\
\text { programmers. }\end{array}$ \\
\hline $\begin{array}{l}\text { Responsive and } \\
\text { flexible }\end{array}$ & $\begin{array}{l}\text { Working with children with autism requires } \\
\text { adaptability during and between therapy sessions. }\end{array}$ \\
\hline $\begin{array}{l}\text { Intuitive control by } \\
\text { a handheld device }\end{array}$ & $\begin{array}{l}\text { Therapists need to be able to direct the robot during } \\
\text { therapy without distracting the child. A small control } \\
\text { device can be concealed. }\end{array}$ \\
\hline
\end{tabular}

Of these requirements, we believe that adaptability is the most important, followed by intuitive control by a handheld device. With adaptability and intuitive control, therapists can begin to use robots in therapy following our approach. Making the interface understandable to therapists is still important, but our current focus is on the two other requirements. Our user interface consists of a design mode and an operation mode. We will discuss these two modes in the following paragraphs. We built the design mode as a simple visual programming environment in order to make it understandable to therapists. Fig. 4 shows the current state of our user interface. 
Although our visual programming environment is simple when compared to other visual programming languages, it has the expressive power of a finite state machine (if/else branching and do-while loops). Users can assemble predefined actions (indicated in Fig. 4 as boxes) and indicate the flow between actions (indicated in Fig. 4 as arrows) to create new choreographies for the robot. Actions include motions, sounds, and facial expressions. In addition to actions, there are user input nodes that allow for branching, shown as a thick border around two of the boxes in Fig. 4. Branch nodes afford some adaptability to changes during a therapy session. Before a therapy session takes place, the therapist can design several sub-choreographies to choose from based on the particular child they will be treating. If one of the sub-choreographies is not eliciting the desired behavior from the child, the therapist can change what the robot is doing at the next branch point.

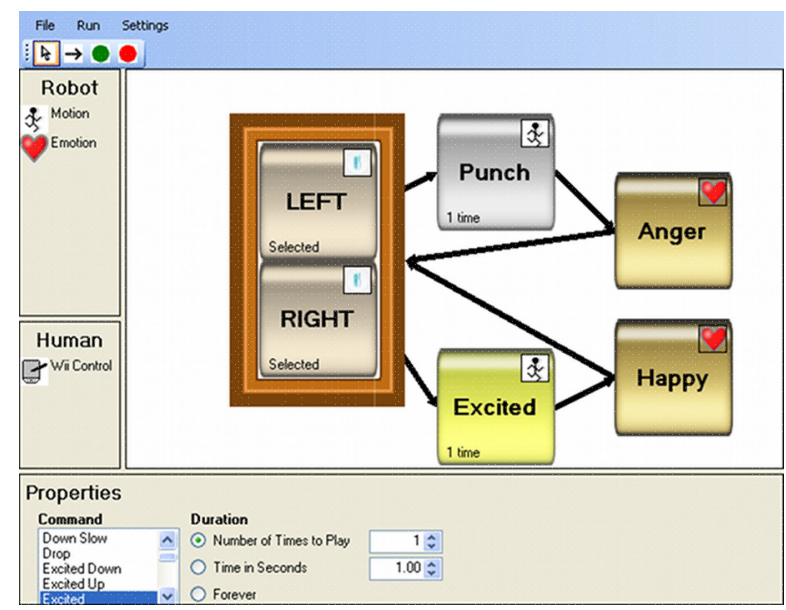

Figure 4. Screenshot of the user interface for designing choreographies with a visual programming paradigm

Therapists direct the robots actions during operation mode at branch points. This is done by pressing buttons on a $\mathrm{Wii}^{\mathrm{TM}}$ Remote control. We chose this remote control because of its small size, simple wireless communication, and a different tactile feel to most of the buttons (this allows therapists to find a particular button without looking at the device). When the program flow comes to a user input node while running in operation mode, the program waits for user input and follows the program flow that corresponds to the user input. To illustrate, Fig. 4 shows a simple choreography with one branch point indicated by a thick rectangular border. Fig. 5 shows a possible interaction between the therapist and robot while using the choreography shown in Fig. 4 in operation mode.

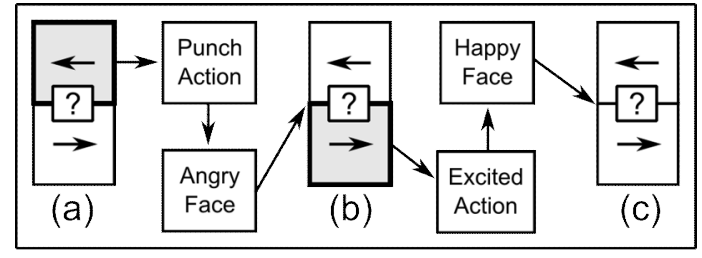

Figure 5. Timeline for a siaple interaction between therapist and robot

At (a), the therapist presses the "left" button and the robot performs the "punch" motion followed by the "anger" facial expression. At (b), the therapist presses the "right" button, and the robot performs the "excited" action followed by the "happy" facial expression. At (c), the choreography returns to the branch point and waits for input. We believe that this type of interaction is a good start toward fulfilling the "intuitive control by a handheld device" requirement. Currently, the therapist is required to memorize the sequence to know which button to push when, but a handheld PDA has been considered to allow the therapist to see the progression of the actions rather than have to memorize them.

\section{Clinical Testing}

Troy was recently used in a clinical trial with typically developing children. Some of the therapeutic activities mentioned in section II.B were evaluated to see what types of reactions Troy would elicit in children and to see how well Troy could carry out these therapies. This trial provided a baseline to evaluate the feasibility of using Troy with children with autism. If the robot were too complicated to use with typical children, then the added complications of using it with children on the autism spectrum would render it unusable.

The first child $\mathrm{A}$ is a 4 year old boy and the second child B is a 3 year old girl. Each child was brought into a 10'x10' therapy room with the robot placed on a table in the middle with chairs placed around it. Two therapists were in the room with the child. One directed the session and controlled the robot. The other worked directly with the child, helping the child perform the actions and preventing him or her from touching Troy. Child A interacted with the therapist and Troy in a turn-taking, imitation protocol. The therapist performed an action, Troy repeated it, and then the child was asked to perform the same action. Actions included raising both arms, pushing a toy truck across the table, pushing a button on a toy giraffe, and hitting a tambourine. Child B also interacted with the robot and the therapist by taking turns playing a xylophone and pushing the button on the toy giraffe. These interactions continued for about 10-15 minutes each.

Through performing these simple studies, we concluded that it is possible to control the robot during a clinical setting. The robot was able to perform useful interactions with the children, and it appeared that the children treated Troy as a social "other." For example, they waited for Troy to push the truck, held the xylophone up for Troy to play it, and watched Troy when they were to imitate what he had just done. When first introduced to Troy, child A appeared to feel anxious, but soon warmed up to Troy during the session. Child B was first surprised by Troy's autonomous movements, but also quickly became comfortable with it. Towards the end of the session, child A seemed more interested in playing with the toy giraffe than in interacting with Troy. The child asked the help of the therapist to play with the toy and largely ignored the robot. As other researchers have noted that children on the autism spectrum are generally more interested in the robots than their typically developing peers [17], we feel that this situation will not happen as much with those with autism. Also, since the goal is to enhance interactions between therapists and the children, if the robot's presence encourages the children to ask help directly from the therapist, this project will be a success.

The children interacted with both the clinicians and the robot, which is encouraging for ongoing studies involving 
children on the autism spectrum. The clinician was able to send commands to the robot via a $\mathrm{Wii}^{\mathrm{TM}}$ remote she held in her hand, often out of sight of the child. Neither child appeared to notice that the clinician was controlling the robot. This could be useful since children with autism may fixate on the remote and want to control the robot themselves. It also demonstrated that with some training the clinicians were able to learn the user interface well enough to control the robot in a clinical setting. These clinical trials validated the possibility of using Troy in a therapy session.

Pilot trials involving two children with autism are underway. These studies are being used to evaluate the children's reactions to Troy and the ability of Troy to encourage child-therapist interactions. Therapeutic activities like the ones explained in section II.B have been tested with both children. Preliminary results show great promise: both children have shown significantly greater interest in Troy than in their typical therapies. They also appeared to attend more to the activities and they interacted more with the therapist than in previous therapy sessions without Troy.

These studies are ongoing; trained therapists continue to use Troy in the clinic to assess its usefulness and develop new methods for achieving clinical benefits. Additional children are being recruited for involvement in these studies. As the studies continue, the level of generalization will also be assessed.

\section{CONCLUSION}

The use of robots in therapy of children with autism holds great promise. Specifications have been outlined to better define the requirements needed for robots to be effective in therapeutic settings. The specifications will not only help evaluate the suitability of a robot for use in autism therapy, but will also guide researchers in their creation of new robots.

The robots that we have created will be evaluated by therapists and tested in actual clinical situations to assess their applicability and analyze what other requirements may need to be met to provide more useful tools for therapy. Other possible robot configurations and requirements will be explored, such as mobile robots, and improvements will continue to be made to the requirements.

\section{ACKNOWLEDGMENT}

The authors wish to acknowledge the support of a Honda Initiation Grant and a Brigham Young University Mentored Environment Grant.

\section{REFERENCES}

[1] C. Kasari, S. Freeman, and T. Paparella, "Joint attention and symbolic play in young children with autism: a randomized controlled intervention study,” J. Child Psychol. Psyc., vol. 47, pp. 611-620, 2006.

[2] B. Robins, K. Dautenhahn, R. Te Boekhorst, and A. Billard, "Robotic Assistants in Therapy and Education of Children with Autism: Can a Small Humanoid Robot Help Encourage Social Interaction Skills?," Universal Access in the Information Society, vol. 4, no. 2, pp. 105-120, Dec. 2005.

[3] B. Robins, N. Otero, E. Ferrari, K. Dautenhahn, "Eliciting Requirements for a Robotic Toy for Children with Autism - Results from User Panels," 16th IEEE International Symposium on Robot and Human Interactive Communication, Jeju, Korea, Aug. 26-29, 2007.
[4] D. Campolo et al., "A novel technological approach towards the early diagnosis of neurodevelopmental disorders," $30^{\text {th }}$ Annual International IEEE EMBS Conference, Vancouver, Canada, 20-24 August 2008, pp. $4875-4878$.

[5] B. Scassellati. Personal communication, Nov. 2008.

[6] B. Robins, P. Dickerson, P. Stribling, and K. Dautenhahn, "RobotMediated Joint Attention in Children with Autism: A Case Study in Robot-Human Interaction," Interaction Studies, vol. 5, no. 2, pp. 161198, 2004.

[7] D. Ricks and M. Colton, "Trends and considerations in robot-assisted autism therapy," 2010 IEEE International Conference on Robotics and Automation, Anchorage, AK, 3-8 May 2010, in press.

[8] K. Dautenhahn and I. Werry, "Towards interactive robots in autism therapy: Background, motivation and challenges," Pragmatics \& Cognition, vol. 12, no. 1, pp. 1-35, 2004.

[9] D. J. Feil-Seifer and M. Mataric, "Robot-Assisted Therapy for Children with Autism Spectrum Disorders," Procs. Conf. on Interaction Design for Children: Children with Special Needs, Chicago, USA, 2008.

[10] G. Bird, J. Leighton, C. Press, and C. Heyes, "Intact Automatic Imitation of Human and Robot Actions in Autism Spectrum Disorders," Proceedings: Biological Sciences, vol. 274, issue 1628, pp. 3027-3031, Oct. 2007.

[11] A. Duquette, F. Michaud, H. Mercier, "Exploring the use of a mobile robot as an imitation agent with children with low-functioning autism," Auton Robot, vol. 24, no. 2, pp. 147-157, 2008.

[12] P. Marti, C. Moderini, L. Giusti, and A. Pollini, "A robotic toy for children with special needs: From requirements to design," 2009 IEEE 11th International Conference on Rehabilitation Robotics, Kyoto, Japan, June 23-26, 2009.

[13] G. Pioggia et al.., "An android for enhancing social skills and emotion recognition in people with autism," IEEE Trans. on Neural Systems and Rehabilitation Engineering, vol. 13, no. 4, pp. 507-15, 2005.

[14] P. De Silva, K. Tadano, A. Saito, S. Lambacher, and M. Higashi, "Therapeutic-assisted robot for children with autism," 2009 IEEE/RSJ International Conference on Intelligent Robots and Systems, St. Louis, USA, Oct. 11-15, 2009.

[15] H. Kozima, C. Nakagawa, and Y. Yasuda, "Interactive robots for communication-care: a case-study in autism therapy," Procs. IEEE ROMAN05, Nashville, USA, 2005.

[16] B. Robins and K. Dautenhahn, "The Role of the Experimenter in HRI Research-a Case Study Evaluation of Children with Autism Interacting with a Robotic Toy," Procs. IEEE RO-MAN06, Hatfield, UK, 2006.

[17] B. Robins, K. Dautenhahn, and J. Dubowski, "Does appearance matter in the interaction of children with autism with a humanoid robot?" Interaction Studies, vol. 7, no. 3, pp. 479-512, 2006.

[18] H. Kozima, and C. Nakagawa, "Interactive robots as facilitators of children's social development," in Mobile Robots towards New Applications, Aleksandar Lazinica, Ed., Vienna: Advanced Robotic Systems, 2006, pp.271-286.

[19] A. R. Tilley and Henry Dreyfuss Associates, The measure of man and woman: Human factors in design. New York: The Whitney Library of Design, 1993.

[20] B. Ingersoll, and L. Schreibman, "Teaching reciprocal imitation skills to young children with autism using a naturalistic behavioral approach: effects on language, pretend play, and joint attention.” J. Autism Dev. Disord., vol. 36, no. 4, pp. 487-505, May 2006.

[21] T. B. Sheridan, Telerobotics, Automation, and Human Supervisory Control. MIT Press, 1992.

[22] M. Yim, "Astronauts Must Program Robots," AAAI 2006 Spring Symposium: To Boldly Go Where No Human-Robot Team Has Gone Before. Menlo Park, California, USA, March 27-29, 2006.

[23] T. R. Green and M. Petre, "Usability Analysis of Visual Programming Environments: A 'Cognitive Dimensions' Framework," J. Visual Lang. Comput., vol. 7, no. 2, pp. 131-174, 1996.

[24] M. A. Goodrich and D. R. Olsen, Jr., "Seven principles of efficient human robot interaction," Proc. IEEE International Conference on Systems, Man, and Cybernetics, vol. 4, pp. 3942-3948, 2003. 